\title{
Endoscopy and oesophagus T149-T157
}

USE OF ARGON BEAMER ELECTROCOAGULATION IN A UK ENDOSCOPY UNIT

Elhasani S, Hodson R M, Tsai H H, Duthie G S, Wedgwood K R Castle Hill Hospital, Cottingham, East Yorkshire, England. HU16 $\underline{5 \mathrm{JO}}$

The argon beamer is a new device that delivers controlled electrocoagulation. This device can be introduced via an endoscope and may aid in haemostasis and tumour debulking in the GI tract. We have evaluated its usefulness in a GI endoscopy unit.

Methods: A prospective audit of the use of the argon beamer (ERBE ICC200) over an 8 month period $(1.10 .95-21.5 .96)$ in a busy endoscopy unit.

Results: A total of 28 therapeutic procedures were carried out using the argon beamer in 18 patients. The therapeutic procedures were for haemostasis (11), tumour debulking (8), angiodysplasia (6), inflammatory polyps (2) and tumour ablation (1). Of those patients whom underwent argon beam coagulation for haemostasis, 2 were for bleeding from oesophageal ulcers (1 malignant), 6 patients had coagulation of the polyp base after snare polypectomy, 1 patient was treated for bleeding after biopsy of a gastric ulcer, 1 patient for bleeding after a papillotomy and 1 patient had a bleeding ampullary cancer. All patients had a successful arrest of bleeding after 1 treatment. 4 patients had treatment for tumour debulking (1 previously stented oesophageal cancer, 1 cardia lesion, 1 rectal, 1 ampullary), requiring a mean of 2 sessions to achieve adequate tumour debulking. 3 patients were successfully treated for angiodysplastic lesions (2 stomach, 1 colonic) after a mean of 2 sessions. 1 patient had a villous tumour of the caecum which was successfully ablated after 1 treatment and 1 patient underwent 2 treatments to successfully eradicate inflammatory polyps in the oesophagus. All operators found the argon beamer easy to use and there were no complications.

Conclusions: Argon beamer electrocoagulation therapy appears to be a safe, effective and versatile tool for the endoscopist. It is valuable as a therapeutic modality for bleeding, tumour debulking and obliteration of angiodysplasia.
T151

COELIAC DISEASE, CLINICAL, ENDOSCOPIC AND HISTOLOGICAL CORRELATION.

A S G Soliman and R Cockel Selly Oak Hospital, Birmingham and Northern General Hospital, Sheffield, U.K.

Endoscopic duodenal biopsy is today the essential procedure for diagnosis of coeliac disease. It is suggested that the macroscopic appearance of the duodenal mucosa seen at endoscopy is a sensitive and specific indicator of histological abnormality.

To assess this claim we reviewed all endoscopies where duodenal biopsies showed coeliac disease during the years 1990-4 in two gastroenterology departments which were familiar with the described mucosal changes. Endoscopic appearance of the mucosa described at the time and the endoscopists' experience were recorded. Most of the examinations were done by standard Olympus fibrescopes.

Coeliac disease was diagnosed histologically in 116 examinations (99 patients - some patients were examined more than once). Only 37 (32\%) examinations were recorded as abnormal at endoscopy. Experienced endoscopists ( $>1000$ examinations) reported 20/53 abnormal, intermediate (500-1000 examinations) $15 / 52$ and novice $(<500) 2 / 11$ - this shows a trend in favour of experience.

Detailed review of 50 cases diagnosed during the years 1992-4 was undertaken to assess whether there were any clinical or investigative features associated with visible mucosal abnormality. Six patients with a "florid" malabsorption syndrome, all had an abnormal appearance. When clinical and laboratory abnormalities were scored together then when the score was greater than three, 19 out of 22 mucosae were visibly abnormal compared with only 7 out of 28 with a score of three or less. $(p<0.01)$

Conclusion : duodenal mucosa is likely to look abnormal when steatorrhoea and malabsorption of multiple nutrients is present and will look normal in most cases with isolated malabsorption. Whilst endoscopically abnormal mucosa should prompt biopsy, the prime indication remains clinical or laboratory suspicion of coeliac disease.

\section{EFFICACY OF PUSH ENTEROSCOPY IN THE} INVESTIGATION OF GASTROINTESTINAL BLOOD LOSS LY.Yiannakou, Colette Hawkins, A.I.Morris*. Gastroenterology Units, St. Thomas' Hospital, London, *The Royal Liverpool University Hospital

Introduction: Push enteroscopy (PE) allows examination, biopsy and therapy of the distal duodenum and jejunum. A major indication of PE is the investigation of GI blood loss when other investigations have been normal, and we have assessed its efficacy in this situation.

Methods: We reviewed case records of 54 consecutive, unselected, patients who underwent $P E$ to investigate occult $(n=38)$ or overt $(n=16)$ gastrointestinal bleeding. 29 patients were male and mean age was 52.2 years (range 17-88). Investigations prior to PE were: OGD (50 patients), colonoscopy (34), small bowel radiology (12), barium enema (14), radioisotope scans (18) and angiography (4). Mean transfusion requirement was 13.7 units (range $(0-120)$. Procedures were performed with an Olympus SIF 10 fibreoptic enteroscope under sedation with midazolam (mean $7.6 \mathrm{mg}$ ). Mean duration of procedure was 24 minutes

Results: All procedures were well tolerated with no complications Causes of GI blood loss were identified in 24 patients (44\%) and these were: angiodysplasia (17 patients), ulceration (3), primary adenocarcinoma (1), metastases (1), ulcerated diverticulosis (1), and Crohn's disease (1). A causitive lesion was identified in $37 \%$ of patients with occult, and $62 \%$ of overt GI blood loss. Endoscopic therapy was given, using an ndYAG laser, to 8 patients with angiodysplasia and one patient with jejunal metastases. All patients showed an initial improvement with cessation of overt bleeding and reduced transfusion requirement. The patient with metastatic disease succumbed from her primary disease within two weeks of treatment. Four patients with angiodysplasia have had no further bleeding (minimum of 8 months follow-up), whilst the other 4 have required iron supplements because of extensive angiodysplasia throughout the GI tract.

Conclusions: PE is safe and well tolerated even in the young and elderly. Causes of GI blood loss are detected in a high proprtion of patients with negative conventional endoscopy and radiology, especially in those with overt bleeding, and effective treatment can be provided.

\section{EXPERIENCE WITH QUALITY OF RINSE WATER IN ENDOSCOPIC DISINFECTION.}

A.Soliman*, P.Finner, S.Lee, C.Hill*, P.Norman \& D.J. Dawson*. Public Health Laboratory Service \& Department of Gastroentrology. * Northern General Hospital, Sheffield.

Infections due to inadequate endoscope decontamination are rare, although cases of Ps.aeruginosa infections have been reported following ERCP related to contaminated automatic washing machines / water bottles. Atypical mycobacterial infection can also occur in immunocompromised patients or lead to misdiagnosis after bronchoscopy

Two Afos(ICU)2 automated washing machines are used in our unit. Routine microbiology checks showed high bacterial count from pre- and post filtration, and the machine bath, $(>1000$ organism $/ \mathrm{ml}$ including pseudomonas, atypical mycobacteria and moulds).

We therefore compared two treatment methods (Promed Systems LTD) to improve water quality. Both incorporated disinfection of the filtration system with peracetic acid (for 60 hours each weekend). One system also had in-line UV disinfection. Weekly samples were taken post-filtration (point A) and from the bath(point B) before and after disinfection cycle in both systems for three months.

Result : ( mean before/after) (bef/aft) (bef/aft) (bef/aft) $\begin{array}{lllll}22^{\circ} \mathrm{C} \text { plate count }(\mathrm{cfu} / \mathrm{ml}) & 2 / 1 & 241 / 2 & 16 / 4 & 167 / 59\end{array}$ $\begin{array}{lllll}\text { pseudomonas count }(\mathrm{cfu} / \mathrm{ml}) & 1 / 0 & 44 / 1 & 4 / 1 & 41 / 23\end{array}$ Mycobacteria and moulds less than $1 / 100 \mathrm{ml}$ in all samples Ps.aeruginosa less than $1 / \mathrm{ml}$ in all samples.

Conclusion : Both systems produced water which was consistently free of mycobacteria, moulds and Ps.aeruginosa and had reduced general bacterial and pseudomonas counts. This was maintained between weekly disinfection cycles. No significant advantage was seen with the addition of UV. The chemical disinfection alone is less costly. 
ARE PHYSICLAN ENDOSCOPISTS AT INCREASED RISK FROM occupational MUSCULOSKELETAL PROBLEMS? A.S. Mee+, J Stratford $^{+}$, J David ${ }^{*}$. Departments of Gastroenterology ${ }^{+}$and Rheumatology ${ }^{\star}$, Royal Berkshire \& Battle Hospitals NHS Trust, Reading, UK.

Introduction Anecdotal reports suggest that endoscopists suffer from a variety of occupation related musculoskeletal problems. To test this hypothesis 165 consultant physician endoscopists and 160 nonendoscoping consultant controls (rheumatologists) were sent a postal questionnaire requesting information on symptoms of neck pain, $\mathrm{X}$ ray documented cervical spondylosis (if known), lateral epicondylitis, thumb extensor tenosynovitis, osteoarthritis of the carpo-metocarpal joint of the thumb and non-disc related low back pain. Endoscopists were asked to quantify approximate career numbers of upper gastrointestinal endoscopies, colonoscopies and ERCP's undertaken, whether or not the weight of the instrument was routinely increased by the use of a teaching arm and the proportion of work using video vs. fibreoptic instruments.

Results There were 156/165 (95\%) evaluable replies from the endoscopists (mean age $46.4 \mathrm{y}$, range 32-66). and $150 / 160$ (94\%) from the rheumatologists (mean age $44.6 \mathrm{y}$, range 30-65). Among the endoscopists $101(65 \%)$ reported one or more musculoskeletal problems compared with $118(79 \%)$ rheumatologists. For endoscopist who reported a problem there was no correlation with their age, type of instrument or use of a teaching arm. Nevertheless, endoscopists performing the greatest number of procedures were significantly more likely to report a problem. For example the career total number of colonoscopies performed by 23 endoscopists reporting lateral epicondylitis ('tennis elbow') was 2830 compared with 2167 colonoscopies in 49 endoscopists with no problem $(p<0.01)$.

Conclusion: There is no overall increased risk of musculoskeletal problems in physician endoscopists compared with non-endoscoping consultant physicians. However those endoscopists reporting a problem performed a significantly greater number of procedures than those without problems.
ONE WEEK OMEPRAZOLE TREATMENT AS A DIAGNOSTIC TEST FOR GASTRO-OESOPHAGEAL REFLUX DISEASE

Eolke Johnsson, Lars Weyvadt", Jan-Helge Solhaug ${ }^{+}$, Håkan Hernqvist $^{++}$, Leif Bengtsson ${ }^{+}$, Department of Surgery, Lund University Hospital, Sweden; Department of Medicine, Holstebro Central Hospital, Denmark"; Department of Surgery, Diakonihjemmet Hospital, Oslo, Norway" and Hässle Läkemedel, Sweden ${ }^{+1}$ (introduced by J. Bennett).

The aim of this study was to evaluate the ability of a one week treatment-test with omeprazole to establish the diagnosis of gastrooesophageal reflux disease. In a prospective, randomized, double-blind Scandinavian multicentre study 188 patients with dyspeptic symptoms including heartburn were enrolled preentry and 160 were randomized to one week treatment of omeprazole $20 \mathrm{mg}$ bid or placebo. Gastrooesophageal reflux disease was defined as reflux oesophagitis SavaryMiller grade II - III at endoscopy or $\mathrm{pH}<4$ exceeding $4 \%$ of the total time at $\mathbf{2 4}$ hour esophageal $\mathrm{pH}$-monitoring and was found in 135 patients. The treatment test was considered positive if the patients symptoms improved during the treatment week compared to the pretreatment day. The sensitivity in diagnosing reflux disease was $71-81 \%$ using omeprazole as a diagnostic test compared to $36-47 \%$ for placebo during treatment days 3-7. The specificity was similar between the two treatment arms during the first days of the study. During the end of the week a larger proportion of the patient's with normal endoscopy and $\mathrm{pH}$-test responded to omeprazole treatment, giving omeprazole lower specificity than placebo. The investigators overall evaluation if the patient was a responder to the test had a sensitivity of $75 \%$ and a specificity of $55 \%$ in the omeprazole treated patients. The corresponding figures in the placebo group were $17 \%$ and $92 \%$, respectively. We conclude that one week of omeprazole treatment is a useful diagnostic test with a high sensitivity that adds to the diagnostic possibilities in patients with reflux disease.
SELECTION OF PATIENTS FOR PRE - LAPAROSCOPIC CHOLECYSTECTOMY ERCP USING "STRICTER CRITERIA" OF JAUNDICE AND DUCTAL STONES ON IMAGING.

TCK Tham, DR Lichtenstein, J Vandervoort, RCK Wong, D Brooks*, J Van Dam, F Ruymann, F Farraye, DL Carr-Locke. Division of Gastroenterology, Brigham \& Women's Hospital, Boston, MA, U.S.A.

When ERCP is performed pre-laparoscopic cholecystectomy in patients with suspected common bile duct (CBD) stones, the detection rate is low (27-50\%). There has been a move towards less pre-operative and more post-operative ERCP except in patients who are jaundiced or who have CBD stones demonstrated on ultrasound scan or CT.

AIM: To assess the accuracy of stricter criteria for pre-operative ERCP in the diagnosis of CBD stones.

METHODS: 1097 laparoscopic cholecystectomies performed in this center from 1993 - 1995 were analyzed for ERCP involvement. A high likelihood for risk of CBD stones was considered an indication for preoperative ERCP and was defined as presence of either bilirubin $>2 \mathrm{mg} \%$, alkaline phosphatase $>150 \mathrm{U} / \mathrm{h}$, present/recent jaundice/pancreatitis, or dilated CBD/stone on scan. The strategy of using "stricter criteria" for pre-operative ERCP which was defined as jaundice and/or demonstrated stones on scan, was then evaluated retrospectively.

RESULTS: 107 (9.8\%) pre-op ERCPs were performed for high likelihood of CBD stones and 34 (32\%) were found to have CBD stones, $1(1 \%)$ had bile crystals, 1 (1\%) CBD stricture, 1 (1\%) PD stricture, 69 (64\%) were normal. ERCP failed in $1(1 \%)$. If the strategy of using a stricter criteria for pre-op ERCP was applied, 54 of the patients would have undergone pre-op ERCP (5\% of all lap choles; $50 \%$ reduction in pre-op ERCP), stones would have been found in 30 (56\%) while 4 with stones would have been missed. 9 other patients had ductal stones found at post-op ERCP. Thus the incidence of stones missed pre-op would be $1.2 \%$.

CONCLUSIONS: If the standard criteria for likelihood of CBD stones is used to select patients for pre-op ERCP, the incidence of CBD stones is low. However if the strategy of stricter criteria is applied, the number of pre-op ERCPs is reduced and the incidence of stones increased while only a minority with stones will be missed.
THE EFFECT OF $\mathrm{pH}$ AND TEMPERATURE STRESS ON FLUID PHASE ENDOCYTOSIS BY HUMAN OESOPHAGEAL EPITHELIUM. W. Hall, D. Hopwood, P. E. Ross. Department of Molecular and Cellular Pathology, Ninewells Hospital and Medical School, Dundee, DD1 9SY.

Aims: Fluid phase endocytosis is a good marker of cellular function and has previously been shown to occur in human oesophagus. This work aimed to determine the effect of alteration of $\mathrm{pH}$ and temperature on the endocytosis of $0.01 \mu \mathrm{m}$ fluorescent microspheres by normal human oesophageal epithelial cells.

Methods: Endoscopic biopsies from normal human oesophagus were subjected to stress by an alteration in either $\mathrm{pH}$ (2-12 using a phosphate/citrate/borate buffer) or temperature $\left(37-55^{\circ} \mathrm{C}\right)$ for $15 \mathrm{mins}$. The biopsies were then placed into organ culture overnight with a solution containing $0.01 \mu \mathrm{m}$ FITC labelled microspheres in Ham's F-10 medium. Following physical disaggregation, the single cell suspensions were analysed using confocal microscopy and flow cytometry.

Results: The effect of stress on fluid phase endocytosis of microspheres was quantitated by flow cytometry. The data shows the mean fluorescence values in arbitrary units of $6-8$ samples.

\begin{tabular}{|l|l|l|l|}
\hline $\mathrm{pH}$ & 3 & 7 & 11 \\
\hline FLUORESCENCE & 33 & 90 & 39 \\
\hline
\end{tabular}

\begin{tabular}{|l|l|l|l|}
\hline TEMPERATURE & $37^{\circ} \mathrm{C}$ & $45^{\circ} \mathrm{C}$ & $55^{\circ} \mathrm{C}$ \\
\hline FLUORESCENCE & 100 & 99 & 110 \\
\hline
\end{tabular}

Conclusions: The alteration of extracellular $\mathrm{pH}$ for a short time causes a persistent alteration in the ability of human oesophageal epithelial cells to endocytose fluorescent microspheres i.e. this has altered cellular function. Elevating temperature does not alter endocytosis, indicating that defence mechanisms exist which are triggered by heat but not by $\mathrm{H}^{+}$ion concentration. This technique will prove useful in elucidating the mechanisms of damage and protection in the human oesophagus. 
FIRST LINE TREATMENT WITH OMEPRAZOLE 10MG OM IS A MORE EFFECTIVE STRATEGY THAN GAVISCON ${ }^{\circledR} 10$ ML QID IN $^{2}$ THE RELIEF OF DYSPEPSIA SYMPTOMS IN PRIMARY CARE.

J. Goves ${ }^{1}$, J.K. Oldring, D. Kerr, E.P. Carr ${ }^{2}$ and M.L. Turbitt ${ }^{2}$ on behalf of a UK study group (introduced by P.D.I. Richardson).

${ }^{1}$ The Enstone Surgery, Enstone Road, Charlbury, Oxon. OX7 3PQ.

${ }^{2}$ Clinical Research Department, Astra Pharmaceuticals Ltd., Home Park, Kings Langley, Herts. WD4 8DH.

Six hundred and seventy four dyspeptic patients with heartburn and/or epigastric pain as their predominant symptom and who had no proven abnormality (e.g. proven peptic ulcer) to explain their symptoms were blindly randomised to either omeprazole $10 \mathrm{mg}$ om or Gaviscon ${ }^{\oplus}$ liquid $10 \mathrm{ml}$ qid for 4 weeks in an open label parallel group study with assessments after 2 and 4 weeks. The primary efficacy variable, percentage of patients with complete symptom relief, was compared between the two groups. Other efficacy variables included the percentage of patients with sufficient symptom control (maximum of 1 day of mild symptoms per week), patients' quality of life (psychological general well -being (PGWB) index and the gastrointestinal subjective rating scale (GSRS)) and patients' subjective assessment of their treatment.

\begin{tabular}{ccccc} 
& \multicolumn{3}{c}{$\begin{array}{c}\text { complete symptom relief } \\
\text { \% at } 2 \text { wks }\end{array}$ \% at 4 wks } & \multicolumn{2}{c}{ sufficient symptom control } \\
\% 2 wks & \% at 4 wks \\
Omeprazole & 27 & 41 & 39 & 50 \\
Gaviscon & 8 & 16 & 17 & 23 \\
p-Values & $<0.0001$ & $<0.0001$ & $<0.0001$ & $<0.0001$
\end{tabular}

Comparisons of quality of life scores between treatments significantly favoured the omeprazole group at 2 and 4 weeks for the PGWB ( $p=$ $0.0002 ; p=0.0009$ respectively) and the GSRS (both $p<0.0001$ ). For subjective assessments of treatments, at 2 and 4 weeks, a significantly greater proportion of patients rated omeprazole to be more effective in symptom relief (both $p=0.0001$ ) and more convenient to use (both $p=$ 0.0001 ) compared to Gaviscon.

This study demonstrates that, compared to Gaviscon $10 \mathrm{ml}$ qid, omeprazole $10 \mathrm{mg}$ om is significantly more effective in the management of dyspepsia symptoms and is the patients' preferred treatment.

\section{Nutrition, small bowel, pancreatico-biliary F158 F158-F172}

OSTEOPENIA IN CROHN'S DISEASE IS LINKED TO POOR

NUTRITION. COULD THIS BE PREVENTED? R.J.Robinson, Research Unit, Leicester General Hospital, Gwendolen Road, Leicester.

Patients with Crohn's disease (CD) are at risk of low bone mineral density (BMD). The aetiology of bone loss is multifactorial with corticosteroid therapy, sex hormone deficiency, disease activity and malnutrition possible contributory factors. The aim of this cross-sectional study was to evaluate nutritonal risk factors for osteopenia in patients with $C D$. Methods 115 patients $(M a l e=48)$ with $C D$ were studied $(M e a n$ age= 40.3 years (SD 13.2). Patients with biochemical osteomalacia, pregnancy, ankylosing spondilitis, medical conditions or medication affecting BMD were excluded. BMD was measured at the lumbar spine (L2-LA) and left hip (femoral neck, trochanter, Ward's triangle) by dual energy X ray absorptiometry (Osteopenia: BMD 1 SD below young adult). Nutritional status was assessed by weight (kg), body mass index $\left(\mathrm{kg} / \mathrm{m}^{2}\right)$, and mid-arm circumference $(\mathrm{cm})$. Subscapular and triceps skinfold thickness $(\mathrm{mm})$ were measured using Holtain Tanner Whitehouse callipers. Each measurement was made 3 times and the mean recorded. Body fat was calculated using the Durnin and Womersley equation.

Results 72 (63\%) patients had osteopenia. Mid arm circumference was significantly lower in patients with osteopenia than patients with normal BMD (28.0 (3.0) vs 29.3 (3.0), $\mathrm{p}=0.04)$. Triceps skinfold thickness was also reduced $(14.9(7.7)$ vs $20.4(7.8), \mathrm{p}=0.0001)$ and $\%$ body fat was significantly lower in the osteopenic patients $(27 \%(8)$ vs $32 \%(6), p=0.001)$. There was no significant difference in weight, BMI or subscapular skinfold thickness. Mean BMD was significantly lower in the 10 patients whose weight fell since age 25 compared to patients whose weight remained stable or increased (Femoral neck -1.69 (1.0) vs -0.55 (1.1) $\mathrm{p}=0.002$, trochanter -1.5 (1.1) vs -0.39 (1.1) $\mathrm{p}=0.004$, Ward's triangle -2.03 (1.1) vs -0.59 (1.1) $p=0.0001$ ). Age, site of disease, current steroid use, lifetime prednisolone dose, previous surgery and \% body fat were entered into a forward stepwise linear regression model. Current prednisolone $(p=0.003)$ and $\%$ body fat $(p=0.01)$ were predictive variables of spine BMD. At the femoral neck $\%$ body fat $(p=0.0001)$, age $(0.0001)$ and lifetime prednisolone dose $(p=0.001)$ were predictive of BMD.

Conclusion Clinical nutritional state is impaired in CD patients with osteopenia, and weight loss in adulthood is associated with low bone density. These are risk factors for osteopenia which can be controlled and should be treated aggressively.
MANIPULATION OF INTESTINAL TRANSIT RATE ALTERS COLONIC LUMINAL $\mathrm{pH}$ AND STOOL SHORT CHAIN FATTY ACID CONCENTRATION. SJ Lewis and KW Heaton. Department of Medicine, Bristol Royal Infirmary, Bristol BS2 8HW.

Populations at low risk of colonic cancer consume large amounts of fibre and starch (fermented by bacteria to short chain fatty acids (SCFA)) and pass acid bulky stools. Traversing the colon SCFA are absorbed and luminal $\mathrm{pH}$ increases to neutral. One SCFA, butyrate, is the colon's main energy source and inhibits malignant transformation in vitro. Low colonic $\mathrm{pH}$ should be associated with high levels of butyrate and thus decreased predisposition to cancer. We set out to test two hypotheses: 1. Altering colonic transit rate alters colonic $\mathrm{pH}$. 2. Distal colonic luminal $\mathrm{pH}$ is correlated with the SCFA (especially butyrate) content of the stools.

13 healthy volunteers took in turn supplements of wheat bran (mean $28.3 \mathrm{~g} /$ day), senna laxative and loperamide, each for nine days with a 2 week washout period. Before and in the last 4 days of each intervention period dietary intake, whole gut transit time (WGTT), stool pH, stool SCFA concentrations (by GLC) and intracolonic $\mathrm{pH}$ (using a radiotelemetry capsule for continuous monitoring) were assessed.

There was no difference between dietary intakes specifically total fibre, NSP or fat at the start and end of each interventional period. pH measurements were similar in the distal colon and stool. WGTT decreased and stool output increased with wheat bran and senna, vice versa with loperamide. Changes in WGTT were least impressive for wheat bran. Baseline stool SCFA concentration correlated with distal colonic $\mathrm{pH}(\mathrm{r}=-0.417, \mathrm{p}=0.01)$ and WGTT $(r=-0.623, \mathrm{p}<0.001)$, similar correlations were seen for baseline stool butyrate (distal $\mathrm{pH} r=0.434$, $\mathrm{p}=0.007$ \& WGTT $\mathrm{r}=0.610, \mathrm{p}<0.001$ ).

\begin{tabular}{|c|c|c|c|c|c|c|c|c|}
\hline \multirow{3}{*}{$\begin{array}{l}\text { Mean } \\
{ }^{*} p<0.05\end{array}$} & \multicolumn{4}{|c|}{ Colonic pH } & \multicolumn{4}{|c|}{ Stool SCFA $(\mu \mathrm{mol} / \mathrm{g})$} \\
\hline & & dle & & & Buty & rate & & \\
\hline & start & end & start & end & start & end & start & end \\
\hline $\begin{array}{l}\text { Wheat bran } \\
\text { Senna }\end{array}$ & $\begin{array}{l}6.8 \\
6.9 \\
6.9\end{array}$ & 6.6 & 7.1 & $\begin{array}{l}6.9^{*} \\
6.7^{*}\end{array}$ & $\begin{array}{l}69 \\
63 \\
69\end{array}$ & $\begin{array}{l}79 \\
193 * \\
23 *\end{array}$ & $\begin{array}{l}443 \\
376 \\
531\end{array}$ & $\begin{array}{l}451 \\
836^{*} \\
345^{*}\end{array}$ \\
\hline
\end{tabular}

There is a relationship between bowel transit rate (diet being constant) and stool $\mathrm{pH}$, stool SCFA concentration and distal colonic $\mathrm{pH}$. This may explain the associations between colonic cancer and dietary fibre, stool output and stool $\mathrm{pH}$, in that stool $\mathrm{pH}$ is a marker for SCFA levels including butyrate.

DIFFERENTIAL FAT METABOLISM IN CACHEXIA OF CHRONIC LIVER DISEASE: AN IN VIVO ${ }^{13} \mathrm{C}$ MRS AND GLC STUDY

EL Thomas', JD Bell², ML Barnard², J Sargentoni², BR Davidson ${ }^{3}$, SD Taylor-Robinson ${ }^{1,2}$

'Gastroenterology and ${ }^{2}$ Robert Steiner MR Unit: Hammersmith Hospital, London W12 ONN and University Department of ${ }^{3}$ Surgery, Royal Free Hospital and School of Medicine, London NW3 2QG

Understanding the metabolic changes controlling weight loss in chronic liver disease may allow rationalisation of dietary regimes which aim to 'build up' patients prior to orthotopic liver transplantation (OLT). Carbon-13 magnetic resonance spectroscopy $\left({ }^{13} \mathrm{C}\right.$ MRS) is a non-invasive method of characterising adipose tissue composition. We examined 22 malnourished patients prior to OLT, using ${ }^{13} \mathrm{C}$ MRS of adipose tissue in vivo. Patients were re-examined 6-8 weeks post OLT. Results were compared to 42 healthy volunteers. Subcutaneous and omental adipose tissue were obtained for comparative GLC analysis from 11 patients at OLT and in 4 volunteers undergoing hernia repair. A non-preferential loss of all major classes of fatty acids were found by ${ }^{13} \mathrm{C}$ MRS prior to OLT. Eight weeks post OLT, subjects showed a considerable increase in body mass with a significant alteration in adipose tissue fatty acid profiles on repeat ${ }^{13} \mathrm{C}$ MRS. ( $18 \%$ increase in saturated fatty acids, $\mathrm{p}<0.01 ; 6 \%$ decrease in unsaturated fatty acids, $p<0.01$ ). This may be secondary to the use of essential polyunsaturated fatty acids for biosynthesis of eicosanoids in the postoperative period.

Conclusion: Application of noninvasive MRS techniques may be of use in the future to design the optimal dietary management of cachexia. 